\title{
Togitare
}

\section{CONSTRUÇÃO E VALIDAÇÃO DE TECNOLOGIA EDUCACIONAL SOBRE CONSUMO DE ÁLCOOL ENTRE UNIVERSITÁRIOS}

\author{
Vanessa Calmont Gusmão Gigante ${ }^{1}$ (i) \\ Rafaela Cruz de Oliveira ${ }^{2}$ (1) \\ Darlisom Sousa Ferreira ${ }^{2}$ (1) \\ Elizabeth Teixeira ${ }^{2}$ (1) \\ Wagner Ferreira Monteiro ${ }^{2}$ (1) \\ Anete Leda de Oliveira Martins ${ }^{2}$ (c) \\ Marcia Helena Machado Nascimento ${ }^{3}$ (i)
}

\section{RESUMO}

Objetivo: construir e validar tecnologia educacional sobre consumo de álcool para universitários.

Método: estudo metodológico, desenvolvido entre junho de 2015 e julho de 2019, em universidade pública do Amazonas, Brasil. Foram realizadas três etapas: construção, validação de conteúdo e validação de aparência. A validação de conteúdo foi realizada em duas fases com 15 profissionais, e a validação de aparência em uma única fase com 91 acadêmicos de quatro cursos da universidade. Resultados: a tecnologia educacional foi nomeada Consumo de Álcool entre Universitários e está disponível no formato de manual impresso e e-book. Apresentou Índice de Validade de Conteúdo de 0,62 na primeira fase e 0,95 na segunda. Quanto à aparência, obteve Índice de Validade Semântica de 0,89.

Considerações finais: o manual mostrou-se válido para utilização entre universitários, e pode contribuir com intervenções educativas nesse âmbito em instituições de ensino superior.

DESCRITORES: Tecnologia Educacional; Educação em Saúde; Educação Superior; Abuso de Álcool; Serviços de Saúde para Estudantes.

\section{CONSTRUCCIÓN Y VALIDACIÓN DE TECNOLOGÍA EDUCATIVA SOBRE EL CONSUMO DE ALCOHOL EN ESTUDIANTES UNIVERSITARIOS}

\section{RESUMEN:}

Objetivo: construir y validar tecnología educativa sobre el consumo de alcohol en estudiantes universitarios. Método: estudio metodológico, desarrollado entre junio de 2015 y julio de 2019, en una universidad pública del Amazonas, Brasil. Se realizaron tres etapas: construcción, validación de contenido y validación de aspecto. La validación de contenido tuvo lugar en dos fases con 15 profesionales, y la validación de aspecto se desarrolló en una única fase con 91 estudiantes de cuatro carreras de la universidad. Resultados: a la tecnología educativa se la denominó Consumo de Alcohol entre Estudiantes Universitarios y está disponible en los formatos de manual impreso y de e-book. Presentó un Índice de Validez de Contenido de 0,62 en la primera fase y de 0,95 en la segunda. En relación al aspecto, obtuvo un Índice de Validez Semántica de 0,89. Consideraciones finales: el manual demostró ser válido para ser usado con estudiantes universitarios, y puede contribuir con intervenciones educativas en este ámbito en instituciones de enseñanza superior.

DESCRIPTORES: Tecnología Educativa; Educación en Salud; Educación Superior; Abuso de Alcohol; Servicios de Salud para Estudiantes. 
As tecnologias educacionais são um conjunto de conhecimentos que possibilitam a preparação, aplicação e acompanhamento de um processo educacional. Podem, assim, mediar ações de educação em saúde, pois são instrumentos que facilitam a relação entre o homem e a educação para que este possa construir o conhecimento ${ }^{(1)}$. Ações de educação em saúde sustentadas por tecnologias capacitam o ser humano para vivenciar de forma mais saudável as fases de sua vida(2).

O período da vida acadêmica é um ciclo de intensas modificações comportamentais e no estilo de vida, além de ser uma fase propensa à aquisição de vícios, o que frequentemente conduz a fatores de risco de doenças. Um dos vícios mais comuns na juventude é a ingestão de álcool. Segundo dados da Organização Mundial de Saúde (OMS), a população brasileira, com idade acima de 15 anos, consome por ano cerca de 7,8 litros de álcool puro per capita, valor acima da média mundial, de 6,4 litros por pessoa ${ }^{(3)}$.

A escolha dessa temática justifica-se, dentre outros aspectos, porque o uso de bebidas alcoólicas pelos jovens está associado a comportamentos de risco, tais como condução de veículos e envolvimento em acidentes de trânsito, relações sexuais desprotegidas, atos violentos e propensão a suicídio, bem como ao desenvolvimento de doenças crônicas ${ }^{(4)}$. Intervenções educativas entre estudantes universitários propiciam mudanças de atitudes e favorecem a diminuição de comportamentos de risco. Tanto intervenções individuais como grupais, mediadas por tecnologias educacionais, são espaços de troca de informações e propiciam reflexões sobre o processo de viver em contexto universitário(5).

Estudo realizado na Argentina identificou um consumo de álcool entre estudantes universitários de 75,3\%(6). Outro estudo que cobriu 24 países da Ásia, África e Américas, com universitários, verificou um consumo de cinco ou mais doses de álcool em eventos em que participam ${ }^{(7)}$.

Múltiplas estratégias podem ser efetivadas, tanto para a promoção da saúde no contexto universitário, como para a reconfiguração dos ambientes saudáveis dentro da comunidade universitária. No entanto, é preciso ir além e efetivar ações educativas, que tenham como eixo principal estilos de vida e condições favoráveis à saúde dos estudantes ${ }^{(5)}$.

Entre as estratégias aplicadas frente aos agravos em saúde, destaca-se a educação em saúde, principalmente quando mediada por materiais educativos que traduzam o conhecimento disponível para uma linguagem clara, e que sejam adequados à realidade do grupo populacional. Sugestões de ações e intervenções de educação em saúde mediadas por tecnologias educacionais são recomendadas em diversos estudos ${ }^{(8)}$.

Pretendeu-se com este estudo despertar a curiosidade e a atenção, bem como contribuir com o autocuidado dos jovens universitários em relação ao consumo de álcool. Assim, objetivou-se construir e validar tecnologia educacional sobre consumo de álcool para universitários.

\section{MÉTODO}

Pesquisa metodológica, conduzida em três etapas: construção, validação de conteúdo e validação de aparência. $O$ referencial teórico-metodológico adotado está pautado nos pressupostos de Pasquali, constituindo-se pelos polos teórico, empírico e analítico. No presente estudo, aplicou-se o polo teórico, voltado à teorização sobre o construto de interesse, o polo empírico, voltado à aplicação na realidade, e o polo analítico, que inclui a 
utilização de testes estatísticos ${ }^{(9-10)}$. Foi realizado entre junho de 2015 e julho de 2019, em uma universidade pública do Amazonas, Brasil, na Escola Superior de Ciências da Saúde.

$\mathrm{Na}$ etapa de construção, a identificação do tema foi pautada numa primeira revisão narrativa, realizada entre junho e julho de 2015, a partir do acesso à Biblioteca Virtual de Saúde, guiada pela pergunta norteadora: Quais as práticas nocivas e prejudiciais à saúde mais comuns entre os estudantes universitários? Dentre as temáticas emergentes, destacou-se o álcool.

Definida a temática foco, procedeu-se à condução de uma revisão integrativa, realizada em junho e julho de 2016, quando foram acessadas as bases de dados LILACS e MEDLINE. Os descritores utilizados foram alcoolismo e autocuidado e palavras-chave universitários e uso de álcool. O produto da revisão integrativa, denominado corpus, foi analisado com apoio do software Atlas.ti, o que propiciou a definição preliminar dos conteúdos a serem abordados na tecnologia educativa. Optou-se inicialmente pela elaboração de um manual no formato impresso por favorecer a organização do conteúdo para a realização da etapa de validação.

A etapa validação de conteúdo ocorreu em duas rodadas, pois na primeira não se atingiu o Índice de Validação de Conteúdo (IVC) mínimo de 0,70. Os especialistas eram das áreas de Enfermagem, Saúde Mental, Tecnologias Educacionais, Psiquiatria, Psicologia, Pedagogia e Design Gráfico, selecionados intencionalmente, por conveniência, na universidade, segundo expertise na temática. Para ampliar esse número, foi utilizada a técnica de bola de neve ${ }^{(11)}$, em que cada participante indicou de um a dois especialistas de outras instituições de ensino superior.

A coleta de dados com os especialistas foi realizada por meio de um instrumento validado(11), entre 2017 e 2018. O instrumento é dividido em duas partes; na primeira, dados de identificação dos especialistas: idade, sexo, área de formação, tempo de formação, função/cargo na instituição em que atua, tempo de atuação, titulação; na segunda, 22 questões específicas, organizadas em três blocos: I- Objetivos, com cinco questões; IIEstrutura e apresentação, com 12 questões; III- Relevância, com cinco questões. Cada enunciado é respondido por meio de escala likert, com valores de 1 a 4 , sendo 1 para totalmente adequado, 2 para adequado, 3 para parcialmente adequado e 4 para inadequado.

Na Figura 1 está demonstrado o fluxo da pesquisa, com as respectivas etapas e desdobramentos realizados entre a produção e a validação. 


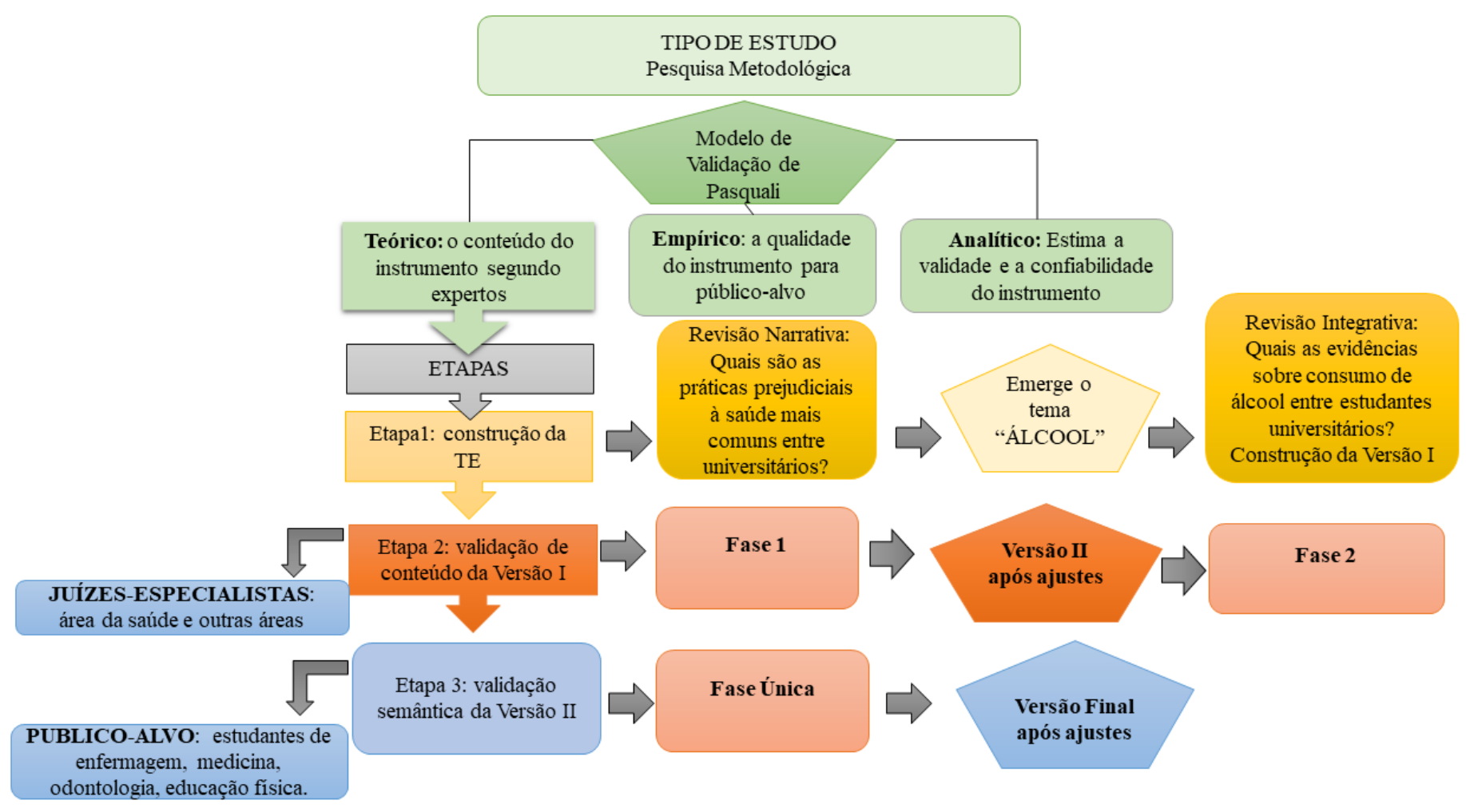

Figura 1 - Fluxograma do método. Manaus, AM, Brasil, 2019

A etapa validação de aparência ocorreu em uma única rodada, pois se atingiu o IVC mínimo de 0,70. Participaram estudantes dos quatro cursos da área da saúde que são oferecidos na universidade: enfermagem, medicina, odontologia e educação física. Todos foram selecionados intencionalmente, por conveniência, a partir da listagem de matriculados no ano de 2018, cursando do $4^{\circ}$ ao $8^{\circ}$ período do curso.

A coleta de dados com o público-alvo foi realizada em 2019, por meio de outro instrumento, também validado(11), dividido em duas partes; na primeira, dados de identificação dos estudantes: período, sexo, idade; na segunda, 26 questões específicas, organizadas em cinco blocos: I - Objetivos, com três questões; II - Organização, com sete questões; III - Estilo da escrita, com seis questões; IV - Aparência, com quatro questões; $\checkmark$ - Motivação, com seis questões. Cada enunciado é respondido por meio de escala likert, com valores de 1 a 4, sendo 1 para totalmente adequado, 2 para adequado, 3 para parcialmente adequado e 4 para inadequado.

Todos os participantes foram convidados a participar por e-mail. Os que responderam, receberam o Termo de Consentimento Livre e Esclarecido. Após aceite formal, receberam o instrumento e a tecnologia educacional em documento digital no formato Portable Document Format (PDF).

Os dados foram armazenados em planilha do Excel. Para a análise estatística das duas rodadas de validação e da rodada única com o público-alvo, estabeleceu-se valor igual ou maior que 0,70 para ser considerado cada item/bloco validado ${ }^{(11)}$.

O estudo foi submetido ao Comitê de Ética em Pesquisa da Universidade do Estado do Amazonas, aprovado conforme parecer número 2.709.552. 


\section{Construção da Tecnologia}

Após leitura dos artigos identificados na revisão integrativa e análise do corpus apoiada pelo software Atlas.ti, foram identificados os seguintes temas: o que é álcool; epidemiologia; padrões de consumo de álcool; o uso de álcool e o universitário; fatores de risco para o uso de álcool; e consequências do uso de álcool. Esse conjunto formou a categoria denominada "Consumo de álcool entre universitários".

Com base na categoria, foram definidos os tópicos principais da primeira versão do manual: o que é álcool, o que a OMS fala sobre o uso nocivo de álcool, o que isto tem a ver comigo, jovem universitário, fatores de risco para o jovem, problemas de saúde, que cuidados devo ter, onde buscar ajuda. $\mathrm{Na}$ construção do manual, adotou-se linguagem clara e objetiva(11) e incluíram-se figuras, posteriormente aperfeiçoadas por uma equipe de design gráfico e ilustrador, além de revisão gramatical do texto. A primeira versão do manual com o título "Consumo Prejudicial de Âlcool entre Universitários" foi estruturada em 13 páginas, com 13 imagens.

\section{Validação de Conteúdo}

Na primeira etapa de validação, dos 15 especialistas, 53,3\% possuíam doutorado, com média de tempo de formação de 19 anos, e 80\% eram docentes em suas áreas de formação/titulação.

Na primeira rodada, o IVC alcançado foi de 0,62. Os participantes apontaram as seguintes sugestões de melhoria: mudanças no layout, representação e cores da capa; correções textuais e redução dos termos técnicos; inclusão de maior representatividade de raças e gêneros nas imagens utilizadas; modificações em títulos; inclusão de imagens contemporâneas.

Após as adaptações, a segunda versão do manual continha 23 páginas e 32 imagens, com as seguintes seções: $O$ que é álcool? Consumo de álcool; $O$ universitário e o álcool; Fatores de risco; Consequências do uso; e Redução de danos, além de duas seções adicionais. Na segunda rodada de validação, obteve-se um IVC total de 0,95 , sendo considerado estatisticamente válido (Tabela 1).

Tabela 1 - Índices de Validação de Conteúdo da primeira e segunda fase de validação segundo blocos do instrumento. Manaus, AM, Brasil, 2019

\begin{tabular}{lcc} 
Itens & \multicolumn{2}{c}{ Índice de Validação de Conteúdo } \\
\cline { 2 - 3 } & 1 $^{\text {a }}$ RODADA & 2 $^{\mathbf{a}}$ RODADA \\
\hline Bloco 1 - Objetivos & 0,49 & 0,93 \\
\hline Bloco 2 - Estrutura e Apresentação & 0,63 & 0,95 \\
\hline Bloco 3 - Relevância & 0,72 & 0,99 \\
\hline IVC FINAL & 0,62 & 0,95
\end{tabular}

Fonte: Autores (2019).

\section{Validação de Aparência}

Dos 91 estudantes que participaram da validação de aparência, 27 eram do curso 
de enfermagem, 36 de medicina, 22 de odontologia e 6 de educação física. A faixa etária variou entre 19 e 21 anos (60,5\%), 22 e 24 anos (28,6\%), 25 e 27 anos $(6,6 \%)$, mais de 30 anos (4,3\%). Quanto ao sexo, $71,4 \%$ feminino e $28,6 \%$ masculino.

Na primeira e única fase foi alcançado um Índice de Validação Semântica (IVS) total de 0,89, acima do mínimo recomendado de 0,70 (Tabela 2).

Tabela 2 - Índices de Validação Semântica da segunda etapa segundo blocos do instrumento. Manaus, AM, Brasil, 2019

\begin{tabular}{lc} 
Itens & $\begin{array}{c}\text { Índice de Validação Semântica } \\
\mathbf{1}^{\mathbf{a}} \text { AVALIAÇÃOO }\end{array}$ \\
\hline Bloco 1 - Objetivos & 0,92 \\
\hline Bloco 2 - Organização & 0,87 \\
\hline Bloco 3 - Estilo da Escrita & 0,89 \\
\hline Bloco 4 - Aparência & 0,87 \\
\hline Bloco 5 - Motivação & 0,9 \\
\hline TOTAL GLOBAL & 0,89
\end{tabular}

Fonte: Autores (2019)

Os estudantes propuseram sugestões, como: diminuição do quantitativo de páginas; mudança do layout da capa; mudança das fontes utilizadas em todo o texto; melhoria da organização do sumário; utilização de uma linguagem mais formal. Após as adaptações, a terceira e última versão do manual apresenta 20 páginas, 10 imagens, mantendo-se as seções da versão anterior.

O manual foi registrado na Fundação Biblioteca Nacional e está disponível no repositório da Universidade do Estado do Amazonas ${ }^{(12)}$.

\section{DISCUSSÃO}

Estudos sobre práticas educativas apontam que os materiais educativos impressos ou digitais contribuem com o processo de comunicação, aumentando a adesão e compreensão do assunto por parte do público a quem são destinados. Há indicativos que orientações escritas têm sido mais efetivas do que as feitas de forma verbal. Também se ressalta a importância de se desenvolver uma tecnologia com linguagem compreensiva à população alvo e de visual atrativo, que estimule a leitura. A disseminação de informação é uma das melhores opções para sensibilizar a comunidade acerca de seus hábitos de vida, e quando voltada para o consumo de álcool entre universitários, um facilitador para capacitação e promoção de saúde desta população(13).

Estudo de revisão sobre o consumo de drogas ilícitas, álcool e tabaco entre universitários identificou que a avaliação das questões de "bebedores problema" entre universitários é discutida como fator preponderante para o entendimento das 
características deles. Destaca-se, no entanto, que os resultados são distintos, de acordo com as universidades brasileiras estudadas. Verificou-se, dentre os resultados, que $21,7 \%$ dos estudantes experimentaram a sensação de perda de controle para pararem de beber e $25,4 \%$ deixaram de fazer o que era esperado em virtude do consumo de álcool(14).

O consumo de álcool entre universitários tem sido alvo de diversos estudos com o objetivo de aprimorar programas de prevenção para esta população, uma vez que o uso exagerado de álcool leva a diversas consequências, a exemplo doenças hepáticas e cardiovasculares, bem como aumenta a incidência de comportamento de risco, como a violência, realização de relações sexuais sem proteção e direção perigosa ${ }^{(15)}$.

As tecnologias educacionais são produtos que emergem de processos concretizados seja a partir da experiência ou da pesquisa. São aplicadas como dispositivos para mediar práticas educativas e têm sido construídas para diferentes públicos: estudantes, comunidade, profissionais. No âmbito dos estudantes universitários, aponta-se um maior quantitativo voltado para o processo ensino-aprendizagem, e menos expressivo para aspectos do cotidiano acadêmico desses estudantes ${ }^{(11)}$.

A efetividade do uso de tecnologias educacionais entre a população universitária, como uma ferramenta abrangente para influenciar a motivação de cessar hábitos prejudiciais à saúde, é um campo novo. Indica-se a necessidade de pesquisas com avaliações mais longas de acompanhamento, em parceria com consultas com o pessoal de saúde que realiza o aconselhamento e apoio no processo de abandono de diferentes usos de drogas e também do consumo de álcool(16), iniciativa que está em fase de implantação na universidade campo desse estudo.

O processo de validação de uma tecnologia educacional é respaldado na premissa de que é fundamental avaliar a legitimidade e a credibilidade do instrumento produzido antes que seja difundido e/ou distribuído ao público-alvo. Igualmente, o estudo de validade de conteúdo é essencial para avaliar a representatividade e clareza de cada item da tecnologia, para que seja aplicável àquela população(10). É necessária validação através de instrumentos que considerem aspectos de conteúdo, estrutura e organização(17).

Os resultados apontaram para uma diversidade de áreas de atuação entre os juízesespecialistas. Estudos que realizaram validação de tecnologias educacionais têm garantido ampla diversidade de profissionais, o que favorece a qualificação da análise e sugestões ${ }^{(18-20)}$.

Destaca-se a importância de terem sido realizadas duas fases de avaliação, pois não se atingiu o valor mínimo estabelecido na primeira. Estudos de validação favorecem a criação de instrumentos confiáveis e atrativos para o público-alvo, subsidiando a disseminação de informações seguras, uma vez que são analisadas por uma gama de profissionais com experiência no assunto(10), que avaliam os conteúdos e a relevância no contexto abordado(20).

As contribuições recebidas, ao serem incorporadas, fortaleceram o escopo técnicocientífico da tecnologia, contribuindo para um maior alcance dos objetivos do constructo, dadas suas expertises e diferentes visões, análises e significações sobre o manual e seu impacto sobre a saúde dos universitários. Dessa maneira, as sugestões foram essenciais para melhoria qualitativa do conteúdo e aparência ${ }^{(21)}$.

Outro destaque é a participação do público-alvo no processo de validação. A inserção do usuário final no processo garante aproximação adequada com esta população, pois somente o público-alvo pode apontar diretamente o que falta para que eles se identifiquem com o material(22). Os universitários, ao criticarem e/ou colaborarem com seu entendimento do que foi escrito e retratado, podem ajudar os autores a refinar a tecnologia, para que fuja de estigmas e imagens pré-concebidas e distantes da realidade desses jovens, estabelecendo-a como dispositivo facilitador de educação e diálogo em saúde.

A partir da validação, identificaram-se pontos a melhorar no manual quanto à 
legibilidade e apresentação. A organização de um guia impresso, no que se refere à sequência do texto, figuras e imagens, organização das mensagens, sinalização de trechos e ideias importantes, uso de cores, espaçamento, e tipo de letra, facilita a legibilidade de materiais educativos ${ }^{(23)}$.

Há uma seção sobre as consequências do uso, por exemplo, em relação ao ato de dirigir. A literatura aponta que o sexo masculino e as idades mais jovens são características que coincidem com aquelas associadas às mortes por acidentes de trânsito(24).

Considerando que o manual foi avaliado como adequado na segunda fase da primeira etapa e na fase única da segunda etapa, emerge como dispositivo para educação em saúde a favor do autocuidado e da saúde dos universitários. Reforça-se o papel da educação em saúde como estratégia de promoção de saúde, e dentre os seus objetivos há que ressaltar o de tornar o usuário capaz de construir um pensamento crítico e também de visualizar e adotar formas alternativas para solucionar problemas enfrentados, oferecendo dicas e informações com vistas à adoção de novos hábitos e condutas de saúde ${ }^{(23)}$. Adotando-se a perspectiva de ser a educação em saúde um dispositivo, tem potencial para o gerenciamento de si, o autogoverno dos corpos e o autocuidado em saúde ${ }^{(24-26)}$.

Outro aspecto relevante da versão final do manual refere-se ao consumo de álcool e a relação com aspectos culturais. Afirma-se que o consumo tem determinantes culturais, e que não devemos banalizar a análise de situações de uso/abuso em que a própria vida dos/das jovens passa a estar em risco devido, entre outros aspectós, à ausência de ações que possam contribuir para o consumo com mais autonomia ${ }^{(27)}$. É nesse sentido que se buscou construir um dispositivo para mediar estratégias de educação em saúde.

Os resultados ressaltaram a importância de tratar do tópico fatores de risco para o consumo de álcool, que tem relação com a liberdade individual que o acesso à universidade oferece, principalmente àqueles estudantes que partem para estudos nos grandes centros, como é o caso no Estado do Amazonas. Esses jovens podem ser influenciados por motivações externas, como amigos e família, e internas, como a necessidade de pertença, curiosidade, ociosidade, prazer e situações estressantes tais como dificuldades financeiras, inúmeras tarefas acadêmicas, competitividade, autonomia e aumento da responsabilidade, privação do convívio familiar, além das incertezas em relação ao seu futuro profissional(28)"

Enfim, a partir das perspectivas indicadas nos processos de validação do manual, reforçando elementos da autonomia, acredita-se que poderá contribuir para a formação de novas matrizes identitárias, bem como a constituição de sujeitos ativos(25). Essa é a perspectiva que se deseja com a veiculação do manual validado neste estudo.

É importante ressaltar como limitação a não participação de estudantes de outras áreas. Optou-se por abordar os estudantes da escola superior de saúde devido à relação direta entre a temática e a referida área dos estudantes.

\section{CONSIDERAÇÕES FINAIS}

O manual, na sua última versão, revelou-se um dispositivo válido e adequado para ser disponibilizado a universitários. As sugestões apontadas nas etapas de validação de conteúdo e aparência indicam a importância de se submeter dispositivos educacionais a processos de validação.

Vislumbra-se que este manual poderá fornecer informações valiosas a esta população, de forma a produzir questionamentos acerca de seus comportamentos, sensibilização e possível redução de danos advindos do uso de álcool. 
Destaca-se também a incorporação, através deste constructo, do uso de materiais educativos baseados em evidências e submetidos a processos de validação de conteúdo e semântica, para mediar práticas de educação em saúde e a sensibilização de universitários, com vistas à aproximação e diálogo com o público-alvo.

\section{REFERÊNCIAS}

1. Paim LMD, Nietsche EA, Lima MGR. História da tecnologia e sua evolução na assistência e no contexto do cuidado de enfermagem. In: Nietsche EA, Teixeira E, Medeiros HP. Tecnologias cuidativoeducacionais: uma possibilidade para o empoderamento do(a) enfermeiro(a)? Porto Alegre: Moriá; 2014. p.17-36.

2. Peres CM, Suzuki KMF, Azevedo-Marques PM de. Recursos tecnológicos de apoio ao ensino na saúde. Medicina (Ribeirão Preto) [Internet]. 2015 [acesso em 10 out 2019]; 48(3). Disponível em: https://www. revistas.usp.br/rmrp/article/view/104303.

3. World Health Organization. Global status report on alcohol and health 2018. Geneve: WHO; 2018.

4. Maciel MED, Vargas D de. Consumo de álcool entre estudantes de enfermagem. Rev Fund Care Online [Internet]. 2017 [acesso em 30 out 2019]; 9(1). Disponível em: http://dx.doi.org/10.9789/2175-5361.2017. v9i1.64-70.

5. Farinha MG, Centurion NB, Braga TBM, Stefanini JR. Rodas de conversa com universitários: prevenção e promoção de saúde. Rev. Nufen: Phenom. Interd. [Internet]. 2019 [acesso em 12 set 2020]; 11(2).

Disponível em: http://pepsic.bvsalud.org/pdf/rnufen/v11n2/a03.pdf.

6. Acosta LD, Fernandez AR, Pillon SC. Social risk factors for alcohol use among adolescents and youth. Rev Latino-Am Enfermagem [Internet]. 2011 [acesso em 20 maio 2020]; 19(Spec No). Disponível em: https://www.scielo.br/pdf/rlae/v19nspe/15.pdf.

7. Peltzer K, Pengpid S. Heavy drinking and social and health factors in university students from 24 low, middle income and emerging economy countries. Community Ment Health J [Internet]. 2016 [acesso em 20 maio 2017]; 52. Disponível em: https://dx.doi.org/10.1007/s10597-015-9925-x.

8. Tossin BR, Souto VT, Terra MG, Siqueira DF de, Mello A de L, Silva AA da. As práticas educativas e o autocuidado: evidências na produção científica da enfermagem. REME Rev Min Enferm [Internet]. 2016 [acesso em 10 out 2019]; 20(e940). Disponível em: http://doi.org/10.5935/1415-2762.20160010.

9. Souza AC de, Alexandre NMC, Guirardello E de B. Propriedades psicométricas na avaliação de instrumentos: avaliação da confiabilidade e da validade. Epidemiol Serv Saúde [Internet]. 2017 [acesso em 10 out 2019]; 26(3). Disponível em: https://doi.org/10.5123/S1679-49742017000300022.

10. Medeiros RK da S, Ferreira Júnior MA, Pinto DP de SR, Vitor AF, Santos VEP, Barichello E. Modelo de validación de contenido de Pasquali en las investigaciones en Enfermería. Rev Enf Ref [Internet]. 2015 [acesso em 14 set 2019]; 4(4). Disponível em: http://dx.doi.org/10.12707/RIV14009.

11. Teixeira E, Mota VMSS. Tecnologias educacionais em foco. São Paulo: Difusão Editora; 2011.

12. Gigante VCG, Ferreira DS, Oliveira RC de, Teixeira E, Monteiro WF. Consumo de álcool entre universitários. [Internet]. Amazonas: Editora UEA; 2020. Disponível em: http://repositorioinstitucional.uea. edu.br//handle/riuea/3041.

13. Barros MSMR de, Costa LS. Perfil do consumo de álcool entre estudantes universitários. SMAD, Rev. Eletrônica Saúde Mental Álcool Drog. [Internet]. 2019 [acesso em 10 out 2019]; 15(1). Disponível em: https://doi.org/10.11606/issn.1806-6976.smad.2019.000353. 
14. Wagner GA, Andrade AG de. Uso de álcool, tabaco e outras drogas entre estudantes universitários brasileiros. Rev. Psiq. Clín [Internet]. 2008 [acesso em 29 set 2019]; 35(suppl.1). Disponível em: http:// www.scielo.br/pdf/rpc/v35s1/a11v35s1.pdf.

15. Rocha EP, Oliveira APP de, Esteves AVF. Validação das tecnologias educacionais na área de Enfermagem: uma revisão integrativa. Scientia Amazonia [Internet]. 2015 [acesso em 10 out 2019]; 4(3). Disponível em: http://scientia-amazonia.org/wp-content/uploads/2016/06/v4-n3-41-47-2015.pdf.

16. Romero-López AM, Portero-de-la-Cruz S, Vaquero-Abellán M. Efetividade na motivação para parar de fumar de uma plataforma web em estudantes universitários. Rev. Latino-Am. Enfermagem [Internet]. 2020 [acesso em 01 out 2020]; 28(e3318). Disponível em: http://dx.doi.org/10.1590/1518-8345.3731.3318.

17. Bomfim E dos S, Slob EMGB, Oliveira BG de, Ribeiro BS, Carmo EA, Santos PHS, et al. Práticas educativas do enfermeiro no cotidiano na Estratégia de Saúde da Família. Rev Saúde Desenvol. [Internet]. 2016 [acesso em 04 out 2020]; 10(5). Disponível em: https://www.uninter.com/revistasaude/index.php/ saudeDesenvolvimento/article/view/512/344.

18. Moreira AP de A, Sabóia VM, Camacho ACLF, Daher DV, Teixeira E. Jogo educativo de administração de medicamentos: um estudo de validação. [Internet]. Rev bras enferm [Internet]. 2014 [acesso em 10 out 2019]; 67(4). Disponível em: https://www.redalyc.org/pdf/2670/267032000005.pdf.

19. Oliveira SC de, Lopes MV de O, Fernandes AFC. Construção e validação de cartilha educativa para alimentação saudável durante a gravidez. Rev Latino-Am Enferm [Internet]. 2014 [acesso em 10 out 2019]; 22(4). Disponível em: http://www.scielo.br/pdf/rlae/v22n4/pt 0104-1169-rlae-22-04-00611.

20. Marinho PML, Campos MP de A, Rodrigues EOL, Gois CFL, Barreto ID de C. Construção e validação de instrumento de avaliação do uso de tecnologias leves em Unidades de Terapia Intensiva. Rev LatinoAm. Enfermagem [Internet]. 2016 [acesso em 22 set 2019]; 24(e2816). Disponível em: http://dx.doi. org/10.1590/1518-8345.1002.2816.

21. Nóbrega M do OS de S, Munhoz RI, Rovarotto J. Sistema de classificação de pacientes em álcool e outras drogas: construção e validação. Rev esc enferm USP [Internet]. 2018 [acesso em 10 out 2019]; 52(e03324). Disponível em: http://dx.doi.org/10.1590/s1980-220x2017020603324.

22. Lima ACMACC, Bezerra K de C, Sousa DM do N, Rocha J de F, Oriá MOB. Construção e validação de cartilha para prevenção da transmissão vertical do HIV. Acta paul enferm [Internet]. 2017 [acesso em 10 out 2019]; 30(2). Disponível em: http://dx.doi.org/10.1590/1982-0194201700028.

23. Santos JEM dos, Brasil W, Moraes KL, Cordeiro JABL, Oliveira GF de, Bernardes C de P, et al. Comprehension of the education handout and health literacy of pacemaker users. Rev bras enferm [Internet]. 2017 [acesso em 15 set 2019]; 70(3). Disponível em: http://dx.doi.org/10.1590/0034-71672016-0336.

24. Moreira MR, Ribeiro JM, Motta CT, Motta JIJ. Mortalidade por acidentes de transporte de trânsito em adolescentes e jovens, Brasil, 1996-2015: cumpriremos o ODS 3.6?. Ciênc. saúde coletiva [lnternet]. 2018 [acesso em 11 out 2020]; 23(9). Disponível em: https://doi.org/10.1590/1413-81232018239.17082018.

25. Ribeiro WA, Andrade M, Cirino HP, Teixeira JM, Martins LM, Mariano E de S. Adolescência, tabaco, álcool e drogas: uma revisão no olhar preventivo da educação em saúde na ESF. Revista Pró-UniverSUS [Internet]. 2018 [acesso em 25 out 2019]; 09(1). Disponível em: http://www.mpgo.mp.br/portal/ arquivos/2018/07/09/10 2742896 Artigo Adolesc\%C3\%AAncia Tabaco Alcool e Drogas uma revis\%C3\%A3o no olhar preventivo da educa\%C3\%A7\%C3\%A3o em sa\%C3\%BAde na ESF 2018. pdf.

26. Soares AN, Souza V de, Santos FBO, Carneiro ACLL, Gazzinelli MF. Dispositivo educação em saúde: reflexões sobre práticas educativas na atenção primária e formação em enfermagem. Texto contextoenferm [Internet]. 2017 [acesso em 22 set 2019]; 26(3). Disponível em: http://dx.doi.org/10.1590/010407072017000260016.

27. Silva RA da, Menezes J de A. Reflexões sobre o uso do álcool entre jovens quilombolas. Psicol. Soc. [Internet]. 2016 [acesso em 01 out 2020]; 28(1). Disponível em: https://doi.org/10.1590/1807- 
28. Soares WD, Barros KS de J, Araujo TP, Finelli LAC, Jones KM. Álcool como mediador social em universitários. Rev Bras Promoç Saúde [Internet]. 2015 [acesso em 22 set 2019]; 28(3). Disponível em: https://periodicos.unifor.br/RBPS/article/view/3247.

\section{COMO REFERENCIAR ESTE ARTIGO:}

Gigante VCG, Oliveira RC de, Ferreira DS, Teixeira E, Monteiro WF, Martins AL de O, et al. Construção e validação de tecnologia educacional sobre consumo de álcool entre universitários. Cogitare enferm. [Internet]. 2021 [acesso em "colocar data de acesso, dia, mês abreviado e ano"]; 26. Disponível em: http://dx.doi.org/10.5380/ ce.v26i0.71208.

Recebido em: 15/01/2020

Aprovado em: 28/10/2020

Editora associada: Susanne Elero Betiolli

Autor Correspondente:

Elizabeth Teixeira

Universidade do Estado do Amazonas - Manaus, AM, Brasil

E-mail: etfelipe@hotmail.com

Contribuição dos autores:

Contribuições substanciais para a concepção ou desenho do estudo; ou a aquisição, análise ou interpretação de dados do estudo - VCGG, RCO, DSF, ET, WFM

Elaboração e revisão crítica do conteúdo intelectual do estudo - VCGG, DSF, ET, ALOM

Aprovação da versão final do estudo a ser publicado - MHMN

Copyright @ 2021 Este é um artigo em acesso aberto distribuído nos termos da Licença Creative Commons Atribuição, que permite o uso irrestrito, a distribuição e reprodução em qualquer meio desde que o artigo original seja devidamente citado. 